ELECTRONIC RESEARCH ANNOUNCEMENTS OF THE AMERICAN MATHEMATICAL SOCIETY

Volume 4, Pages 56-62 (September 14, 1998)

S $1079-6762(98) 00048-1$

\title{
ASYMPTOTIC RESULTS FOR SUPER-BROWNIAN MOTIONS AND SEMILINEAR DIFFERENTIAL EQUATIONS
}

\author{
TZONG-YOW LEE
}

(Communicated by Mark Freidlin)

\begin{abstract}
Limit laws for three-dimensional super-Brownian motion are derived, conditioned on survival up to a large time. A large deviation principle is proved for the joint behavior of occupation times and their difference. These are done via analyzing the generating function and exploiting a connection between probability and differential/integral equations.
\end{abstract}

\section{INTRODUCTION AND STATEMENT OF RESULTS}

We study occupation time limit theorems for the three-dimensional super-Brownian motions (super-BM) and related processes. This is done by analyzing cumulant generating functions which satisfy some integral equations. In the case of super-BM the integral equation is equivalent to a semilinear PDE.

A sample path, $\left(\mu_{t}(d x) ; t \geq 0\right)$, of the super-BM, is a path of nonnegative Radon measures on $\mathbf{R}^{d}$. When the initial $\mu_{0}(d x)$ is $\mu_{0}=\nu$ we denote by $P_{\nu}$ and $E_{\nu}$ the corresponding probability measure and expectation, respectively. We will omit writing the initial measure in the subscript when it is the Lebesgue measure. For a construction of the processes see, for example, [D], [Dy], [I1].

We now state a property of the process $P$ that is particularly important to our study. For a nonpositive integrable function $\varphi$ define the $\varphi$-occupation time $D_{\varphi, T}$ (a random variable), by

$$
D_{\varphi, T}=\int_{0}^{T} \int_{\mathbf{R}^{d}} \varphi(x) \mu_{t}(d x) d t .
$$

The following connection with differential equations and integral equations is known for the cumulant generating function:

$$
E\left\{\exp D_{\varphi, T}\right\}=\exp \left(\int_{\mathbf{R}^{d}} v(T, x ; \varphi) d x\right), \quad T \geq 0,
$$

where $v(t, x ; \varphi)$ is the solution of

$$
\begin{cases}\frac{\partial v(t, x)}{\partial t} & =\Delta v+v^{2}+\varphi \quad \text { in } t>0, x \in \mathbf{R}^{d}, \\ v(0) & =0, x \in \mathbf{R}^{d} .\end{cases}
$$

Received by the editors April 15, 1998.

1991 Mathematics Subject Classification. Primary 60B12, 60F10; Secondary 60F05, 60J15.

Key words and phrases. Large deviations, occupation time, measure-valued process, branching Brownian motion, semilinear PDE, asymptotics. 
Note that the use of the Laplacian, as opposed to half of the Laplacian, indicates that the underlying Brownian motion is being run at twice of the standard speed.

In order to introduce integral equations let us define the heat kernel and associated operators:

$$
\begin{aligned}
p(t, x) & =(4 \pi t)^{-3 / 2} e^{-|x|^{2} / 4 t}, \\
\int & =\int_{\mathbf{R}^{3}} \\
(A u)(t, x) & =\int_{0}^{t} \int p(t-s, x-y) u(s, y) d y d s, \\
(B h)(x) & =\int\left(\int_{0}^{\infty} p(s, x-y) d s\right) h(y) d y, \\
\|f\| & =\|f\|_{2}=\left(\int f(x)^{2} d x\right)^{1 / 2} .
\end{aligned}
$$

Formally, operator $A$ is inverse to the heat operator $\partial_{t}-\Delta$ and $B$ is inverse to $-\Delta$ in suitable spaces of functions $u(t, x)$ and $h(x)$. The function $v$ in (1.2) is also the solution of the integral equation

$$
v=A\left(v^{2}+\varphi\right) .
$$

The lack of interaction in the super-BM makes formulas (1.2)-(1.4) easy to understand. The building block is the case when the initial is a Dirac delta $\delta_{x}$ measure at $x$. For this case the shorthands $P_{x}$ for the probability measure and $E_{x}$ for the expectation will be used. The simple form (1.2) of the generating function follows easily from the building-block case and the inherent independence property (the lack of interaction) of the super-BM.

For three or higher dimensions, Iscoe has proved (Theorem 1 in [I2]) the strong law that, as $T \rightarrow \infty$, the empirical measure $(1 / T) \int_{0}^{T} \mu_{s}(d x) d s$ converges (in the vague topology) with $P$-probability one to Lebesgue measure. When the space dimension is 2 or less, the law of large numbers fails. For critical branching Brownian motions, which is a particle analogue, Cox and Griffeath [CG] investigate the large deviations from this central tendency. Their results show exponential decay of (large deviation) tail probabilities in 5 or more space dimensions and slower than exponential decay of tail probabilities in 3 and 4 space dimensions. The large deviation rate functions have been studied further [IL], [LR], [DR]. Our interest in this note is in the more detailed behavior of the 3-dimensional case. The problem will be approached by estimating the cumulant generating function, in contrast with estimating cumulants $[\mathrm{CG}],[\mathrm{DR}]$.

It is known that equation (1.4) with $\varphi$ replaced by $\delta_{0}$, exists up to a positive blowup time $t^{*}[\mathrm{IL}]$. That is, the mild solution of (1.3) exists.

Let $\varphi$ be integrable with $\int \varphi d x=1$. It is worked out in [IL] that

$$
c^{2} v\left(c^{2} t, c x ; \varphi\right) \rightarrow v\left(t, x ; \delta_{0}\right)
$$

as $c \rightarrow \infty$.

In the above pointwise convergence it is adequate to think that $x$ is not the origin and before blow-up time. Such convention, adopted throughout the paper, saves us from speaking of convergence to infinity.

One major result in this note is a refined limit: 
Theorem 1.1. Let $\varphi, \xi$ be continuous and compactly supported and $\int \xi d x=0$. Let $z=\int \varphi d x+\|B \xi\|^{2}$. Then

$$
v(t, x \mid c)=c^{2} v\left(c^{2} t, c x ; \varphi+c \xi\right) \rightarrow v\left(t, x ; z \delta_{0}\right)
$$

as $c \rightarrow \infty$.

A probabilistic basis for Theorem 1.1 is as follows. Fix $t>0$ and $x$ not the origin and consider large parameter $c$. A super-BM, initially the $\delta_{c x}$ measure, will ever charge the unit ball with probability of order $c^{-2}$. More precisely, the probability is asymptotically $2(c|x|)^{-2}$ for all bounded domains, not just for the unit ball. Conditioned on charging, the total charge (occupation time) up to time $c^{2} t$ is of order $c$. Furthermore, the difference of charge to the right half-ball (the first coordinate $\left.x_{1}>0\right)$ and to the left half-ball $\left(x_{1}<0\right)$ is of order $c^{1 / 2}$. So, we use the correct normalization of dividing the total occupation time by $c$ and the difference by $c^{1 / 2}$. From such probabilistic thinking (see [L] for example), we anticipate the weak convergence (convergence-in-distribution) result:

Conditioned on charging, the normalized occupation time and the difference converges in distribution to a nondegenerate random vector as c tends to infinity. Moreover, the normalized difference, conditioned that the normalized total occupation time equals $a>0$, converges in distribution to a normal distribution with mean 0 and a variance proportional to a (as can be guessed from the central limit theorem).

The above probabilistic reasoning uses the Brownian scaling and the central limit theorem. The choice of the particular $\varphi, \xi$ (the indicator of the unit ball and the difference of the indicator of the right half-ball and the left half-ball) is used only as an easy-to-visualize example and can be arbitrary.

Our Theorem 1.1 is motivated by the above weak convergence result. More precisely, Theorem 1.1 states that the moment generating function converges, which is sufficient, but not necessary at all for weak convergence. The stronger statement of Theorem 1.1 is however crucial for deriving the large deviation result. Let us summarize the weak convergence result as

Corollary 1.2. Consider $P_{c x}, x$ not the origin. Let $\varphi \geq 0$ be compactly supported and $\int \varphi d x>0$. Then the following hold as $c \rightarrow \infty$.

(i) Conditioned on $D_{\varphi}=\sup _{s>0} D_{\varphi, s}>0$, the normalized $c^{-1} D_{\varphi, c^{2} t}$ converges to a nondegenerate probability distribution on $(0, \infty)$. The limit distribution has the moment generating function $g$,

$$
g(\alpha)=v\left(t, x ; \alpha \int \varphi d x\right) .
$$

(ii) Conditioned on $c^{-1} D_{\varphi, c^{2} t}=a>0$, the normalized $c^{-1 / 2} D_{\xi, c^{2} t}$ converges to a normal distribution of mean 0 and variance $2\|B \xi\|^{2} /\left(\int \varphi d x\right)$.

Theorem 1.1, together with the connection (1.2) with cumulant generating functions then enables us to apply the Gartner-Ellis theorem to establish a large deviation theorem. We now give the rate function and then state the large deviation result.

$$
\left\{\begin{array}{l}
\Lambda_{3}(\theta) \equiv \begin{cases}\int_{\mathbf{R}^{3}} v\left(1, x ; \theta \delta_{0}\right) d x & \text { if }-\infty<\theta<\left(t^{*}\right)^{1 / 2} \\
+\infty & \text { otherwise, }\end{cases} \\
K_{3}(a, b) \equiv \sup _{\alpha, \beta \in \mathbf{R}}\left[a \alpha+b \beta-\Lambda_{3}\left(\alpha \int \varphi d x+\beta^{2}\|B \xi\|^{2}\right)\right] .
\end{array}\right.
$$


Theorem 1.3. Consider the super-BM process $P$ (that is, initially the Lebesgue measure). Suppose that $\varphi, \xi$ are continuous and compactly supported and $\int \xi d x=0$. Let $W_{\varphi, T}$ be the average occupation time:

$$
W_{\varphi, T}=\frac{1}{T} D_{\varphi, T}
$$

Then $\left\{\left(W_{\varphi, T}, T^{1 / 4} W_{\xi, T}\right), T^{1 / 2}\right\}$ is a large deviation system with rate function $K_{3}$.

As long as the connection, such as (1.2)-(1.4) exists between integral/differential equations and the probability theory, it is clear that various techniques from these fields can be brought together to attack the problem. The analytic result for equations is interesting in its own right. Our proof method is based mostly on the comparison principle (maximum principle) for equations. The method reveals that the mathematical result goes somewhat beyond probability interpretations known currently. By this we mean that no probabilistic interpretation is known at present for some of the integral/differential equations that are subject to the same technique. For example, let us replace the quadratic nonlinearity $v^{2}$ by $|v|^{p}, p>1$. The problem yields to the same technique of proof. There is however no simple probabilistic meaning to the case $p>2$.

In order to understand the result better one can look at fractional dimensions as well. This can be done by replacing the Laplacian with the Bessel operator. With the quadratic nonlinearity $v^{2}$, we then can see that qualitatively similar results (as in Theorems 1.1 and 1.3) hold for $2<d<4$. So the investigated phenomenon is common to an interval of dimensions, as opposed to an isolated dimension. Another generalization is to replace BM with stable processes [DR].

As the results apply to general $\varphi, \xi$ functions, they can be extended to a functional level. In the case of weak convergence this is ensured by the Cramer-Wold theorem. In the case of large deviations such higher level results can be obtained by, for example, the Dawson-Gartner projection theorem [DG]. The large deviation principle for the difference of occupation times alone was already carefully established at a functional level in [DR] by analyzing cumulants.

In order to help interested readers pursue further we will prove Theorems 1.1 and 1.3 in a manner readily applicable to various generalizations. For example, the technique of this note yields the following result for super-Bessel processes with fractional dimension $d$. Let the nonlinearity exponent be $p$ in (1.3). In the regime $\frac{2}{p-1}<d<\frac{2}{p-1}+2$, we see that qualitatively same results as Theorems 1.1 and 1.3 hold. The correct exponents depend, of course, on $p, d$. For example, in Theorem 1.3 the exponent $1 / 4$ of $T^{1 / 4}$ now becomes $1-\frac{d(p-1)}{2 p}$. The exponent $1 / 2$ of $T^{1 / 2}$ should be $\left(\frac{d}{2}-\frac{1}{p-1}\right)$. In the corollary, the normalization constant $c^{1 / 2}$ for the charge difference should be $c^{\left(\frac{d}{2 p}-\frac{1}{p-1}\right)}$ and $\|B \xi\|^{2}$ should be $\int|B \xi|^{p} d x$. If one is only interested in integer dimensions, notice that all except $p=1+\frac{2}{m}$ with positive integer $m$ admit two integer dimensions. Theorem 1.3, for example, concerns the case $p=2$, hence $m=1$ and $d=3$ is the only integer dimension.

When $1<p \leq 2$, a connection between cumulant generating functions and differential equations like (1.3) exists. The corresponding PDE result translates into a large deviation principle like Theorem 1.3.

A challenging problem is to prove the counterpart of Theorem 1.3 for the threedimensional voter model. It is proved that the normalization constants are the same for occupation times [BCG]. One naturally anticipates that the same (as 
the super-BM) normalization constants hold also for the difference of occupation times and that a large deviation principle like Theorem 1.3 holds. This remains to be done. Preliminary calculation reveals that some crucial arguments from our approach extend to the four-dimensional super-BM as well; many details however need to be worked out.

\section{OUtLine OF THE PROOF}

For an integrable function $u$ and a positive number $c$ denote by $u_{c}$ the function $x \mapsto c^{3} u(c x)$. Recall from Theorem 1.1 the definition of $v(t, x \mid c)$ and fix arbitrary $\varphi, \xi$. A simple rescaling yields

$$
v(t, x \mid c)=c^{2} v\left(c^{2} t, c x ; c^{-1} \varphi+c^{-1 / 2} \xi\right)=v\left(t, x ; \varphi_{c}+c^{1 / 2} \xi_{c}\right) .
$$

Comparing Theorem 1.1 with the known result [IL] that

$$
v\left(t, x ; \varphi_{c}\right) \rightarrow v\left(t, x ;\left(\int \varphi(x) d x\right) \delta_{0}\right)
$$

as $c \rightarrow \infty$, we see that the $c^{1 / 2} \xi_{c}$ term on the right-hand side of (2.1) contributes to the limit by $\|B \xi\|^{2}$, added to $\left(\int \varphi(x) d x\right)$ to account for the number $z$ in Theorem 1.1. This motivates us to use

$$
w(t, x \mid c)=v(t, x \mid c)-c^{1 / 2} A \xi_{c}
$$

because the equation satisfied by $w$ will no longer have the $c^{1 / 2} \xi_{c}$ term. Precisely, the equation of $w$ is

$$
w=A\left[w^{2}+2 w\left(c^{1 / 2} A \xi_{c}\right)+\left(c^{1 / 2} A \xi_{c}\right)^{2}+\varphi_{c}\right] .
$$

This integral equation clearly shows what is going on since an elementary heatkernel calculation shows for $t>0$ that

$$
\lim _{c \rightarrow \infty} \int\left(c^{1 / 2} A \xi_{c}\right)(t, x)^{2} d x=\|B \xi\|^{2},
$$

which is exactly what we desire to get. The heat-kernel calculation also gives us two other ingredients, (2.6) and (2.7) as follows, of our approach.

$$
\lim _{c \rightarrow \infty} \int\left|\left(c^{1 / 2} A \xi_{c}\right)(t, x)\right|^{q} d x=0
$$

if $\frac{3}{2}<q<2$. And

$$
\lim _{c \rightarrow \infty}\left(c^{1 / 2} A \xi_{c}\right)(t, x)=0 .
$$

Now, back to the equation (2.4) of $w$. In view of (2.5), (2.7) and the definition (2.3) of $w$, Theorem 1.1 requires to prove that

$$
\lim _{c \rightarrow \infty} \int\left(w c^{1 / 2} A \xi_{c}\right)(t, x) d x=0 .
$$

Let $\alpha=w(t, x), \beta=\left(c^{1 / 2} A \xi_{c}\right)(t, x)$. We obtain the crucial limit result (2.8) via the estimate

$$
|2 \alpha \beta| \leq \epsilon \alpha^{r}+b \beta^{q},
$$

where $q, r$ is a pair of conjugate exponents and $\epsilon>0, b=2^{q} q^{-1}(r \epsilon)^{-q / r}$. Let $s>0$ be fixed and less than the blow-up time of $v\left(t, x ; z \delta_{0}\right)$. Because of $(2.6)$ we will use 
$\frac{3}{2}<q<2$, thus $3>r>2$. There then exist $\epsilon_{s, r}$ and $z^{*}>z$ such that for all $\epsilon<\epsilon_{s, r}$ the equation

$$
f=A\left[f^{2}+\epsilon|f|^{r}+z^{*} \delta_{0}\right]
$$

has $L^{2}\left(\mathbf{R}^{3}\right)$ solution up to time $s$. This is because $\left|v\left(s, x ; z \delta_{0}\right)\right|^{r}, r<3$ is integrable. In fact, $v\left(s, x ; z \delta_{0}\right)$ is exactly of order $|x|^{-1}$, the Green's function, near the origin and decays at least exponentially fast near infinity. These behaviors are derived via the comparison (maximum) principle. We are now in the position of a finishing touch. The existence of $f$ in (2.10) and the limit result (2.6) imply that the equation

$$
g=A\left[g^{2}+\epsilon|g|^{r}+b\left|\left(c^{1 / 2} A \xi_{c}\right)(t, x)\right|^{q}+\left(c^{1 / 2} A \xi_{c}\right)^{2}+\varphi_{c}\right]
$$

has $L^{2}\left(\mathbf{R}^{3}\right)$ solution up to time $s$. And the solution $g$ dominates $w$ of equation (2.4). These follow again from the comparison principle, using the fact that $z=$ $\int \varphi d x+\|B \xi\|^{2}<z^{*}$ and the limits (2.5), (2.6) and (2.9). Letting $c$ tend to infinity in (2.11), we see that (2.6) and (2.5) account for the appearance of the $z \delta_{0}$ term in Theorem 1.1. The fuction $v$ of equation (1.4) with $\varphi=z \delta_{0}$ is arrived at upon letting $\epsilon$ tend to 0 . So we conclude

$$
\limsup _{c \rightarrow \infty} v(t, x \mid c) \leq v\left(t, x ; z \delta_{0}\right) .
$$

The other direction of inequality is similarly proved; it is in fact easier because instead of (2.10) we look at

$$
f=A\left[f^{2}-\epsilon|f|^{r}+z_{*} \delta_{0}\right] .
$$

The difference is the sign in front of $\epsilon|f|^{r}$ and $z_{*}<z$. The existence of solution of (2.13) follows from that of $v\left(s, x ; z \delta_{0}\right)$ right away.

The discussion above outlines all arguments for proving Theorem 1.1. The proof of Theorem 1.3 does not require any new idea. When establishing (2.12), we also get

$$
\limsup _{c \rightarrow \infty} \int v(t, x \mid c) d x \leq \int v\left(t, x ; z \delta_{0}\right) d x=\Lambda_{3}(z) .
$$

By the probability-PDE connection given in (1.2) and (1.3), this translates into a limit result for the cumulant generating function of the occupation times. The limit is smooth; see [IL]. So the Gartner-Ellis theorem concludes that the large deviation rate is the Legendre transform of the limit of the cumulant generating function, ending Theorem 1.3.

\section{ACKNOWLEDGEMENT}

We would like to thank I. Iscoe, W.-M. Ni, B. Remillard and J. Rosen for useful discussions.

\section{REFERENCES}

[BCG] Bramson, M., Cox, J.T. and Griffeath, D. (1988) Occupation time large deviations of the voter model. Probab. Th. Rel. Fields 77, 401-413. MR 89c:60116

[CG] Cox, J.T. and Griffeath, D. (1985) Occupation times for critical branching Brownian motions. Ann. Probab. 13, 1108-1132. MR 87h:60102

[D] Dawson, D. (1977) The critical measure diffusion process. Z. Wahrsch. Verw. Gebiete 40, 125-145. MR 57:17857

[DG] Dawson, D., and Gartner, J. (1987) Large deviations for McKean-Vlasov limit of weakly interacting diffusions. Stochastics, 20, 247-308. MR 89c:60092 
[DR] Deuschel, J.-D. and Rosen, J. (1998) Occupation time large deviations for critical branching Brownian motion, super-Brownian motion and related processes. Ann. Probab. 26, no. 2, 602-643. CMP 98:13

[Dy] Dynkin, E. B. (1989) Superprocesses and their linear additive functionals. Trans. Amer. Math. Soc., 314, 255-282. MR 89k:60124

[I1] Iscoe, I. (1986) A weighted occupation time for a class of measure-valued branching processes. Z. Wahr. verw. Gebiete. 71, 85-116. MR 87c:60070

[I2] Iscoe, I. (1986) Ergodic theory and a local occupation time for measure-valued critical branching Brownian motion. Stochastics, 18, 197-243. MR 88a:60148

[IL] Iscoe, I. and Lee, T.-Y. (1993) Large deviations for occupation times of measure-valued branching Brownian motions. Stochastics and Stochastic Reports, 45, 177-209. MR 96a:60027

[L] Lee, T.-Y. (1990) Some limit theorems for critical branching Bessel processes and related semilinear differential equations. Probab. Th. Rel. Fields, 84, 505-520. MR 91e:60245

[LR] Lee, T.-Y. and Remillard, B. (1995) Large deviations for the three-dimensional superBrownian motion. Ann. Probab. 23, 1755-1771. MR 96m:60067

University of Maryland, College Park, MD

E-mail address: tyl@math.umd.edu 